\title{
GCU
}

Glasgow Caledonian

University

University for the Common Good

\section{Modelling the impact of different tuberculosis control interventions on the prevalence of tuberculosis in an overcrowded prison}

Naning, Herlianna; Al-Darraji, Haider Abdulrazzaq Abed; McDonald, Scott; Ismail, Noor Azina; Kamarulzaman, Adeeba

Published in:

Asia-Pacific Journal of Public Health

DOI:

$10.1177 / 1010539518757229$

Publication date:

2018

Document Version

Author accepted manuscript

Link to publication in ResearchOnline

Citation for published version (Harvard):

Naning, H, Al-Darraji, HAA, McDonald, S, Ismail, NA \& Kamarulzaman, A 2018, 'Modelling the impact of different tuberculosis control interventions on the prevalence of tuberculosis in an overcrowded prison', Asia-Pacific Journal of Public Health, vol. 30, no. 3, pp. 235-243. https://doi.org/10.1177/1010539518757229

\section{General rights}

Copyright and moral rights for the publications made accessible in the public portal are retained by the authors and/or other copyright owners and it is a condition of accessing publications that users recognise and abide by the legal requirements associated with these rights.

Take down policy

If you believe that this document breaches copyright please view our takedown policy at https://edshare.gcu.ac.uk/id/eprint/5179 for details

of how to contact us. 


\title{
Modelling the Impact of Different Tuberculosis Control Interventions on the Prevalence of
} Tuberculosis in an Overcrowded Prison

\author{
Authors \\ Herlianna Naning, 1; Haider Abdulrazzaq Abed Al-Darraji, 1,2; Scott McDonald, 1,3,4; Noor Azina \\ Ismail, 5; and Adeeba Kamarulzaman, 1 \\ 1 Centre of Excellence for Research in AIDS (CERiA), Faculty of Medicine, University of Malaya, Kuala Lumpur, Malaysia \\ 2 Centre for International Health, Department of Preventive and Social Medicine, University of Otago School of Medicine, University of \\ Otago, Dunedin, New Zealand \\ 3 Centre for Infectious Disease Control, National Institute for Public Health and the Environment, Bilthoven, Netherlands \\ 4 School of Health \& Life Sciences, Glasgow Caledonian University, Glasgow, Scotland \\ 5 Department of Applied Statistics, Faculty of Economics and Administration, University of Malaya, Kuala Lumpur, Malaysia
}

\begin{abstract}
The aim of this study was to simulate the effects of tuberculosis (TB) treatment strategies interventions in an overcrowded and poorly ventilated prison with both high ( 5 months) and low ( 3 years) turnover of inmates against improved environmental conditions. We used a deterministic transmission model to simulate the effects of treatment of latent TB infection and active TB, or the combination of both treatment strategies. Without any intervention, the TB prevalence is estimated to increase to $8.8 \%$ for a prison with low turnover of inmates but modestly stabilize at $5.8 \%$ for highturnover prisons in a 10-year period. Reducing overcrowding from 6 to 4 inmates per housing cell and increasing the ventilation rate from 2 to 12 air changes per hour combined with any treatment strategy would further reduce the TB prevalence to as low as $0.98 \%$ for a prison with low inmate turnover.
\end{abstract}

Keywords: Malaysia, overcrowded prisons, tuberculosis, LTBI, inmates 


\section{Background}

Tuberculosis (TB) has overtaken HIV infection as the number one global killer from infectious diseases, with an overall 1.7 million TB-related deaths reported in 2016, despite accessibility of TB treatment.1 In 2016, the World Health Organization (WHO) estimated a total of 10.4 million incident TB cases globally, $10 \%$ of whom were living with HIV infection. The burden of TB is highest in the South-East Asian and African regions, which accounted for 70\% of the global TB incidence in 2016.1

In Malaysia, TB is the second most frequently reported communicable disease (after dengue fever) and is the leading cause of death from communicable disease, with a mortality rate of 5.56 per 100 000 persons. 2 In 2013, the prevalence of active TB was estimated at between $0.06 \%$ and $0.23 \%$ of the general population. 3 In recent years, Malaysia has witnessed an increase in the incidence of TB, from 65 cases per 100000 in 2000 to 92 per 100000 in 2016.1

Closed settings, including prisons, present special challenges to national TB control programs. Prisons are concentrated populations of individuals at high risk of TB, such as people who use drugs and/or alcohol, the homeless, people with a history of past incarceration, and people with preexisting comorbidities. Poor environmental conditions, such as overcrowding and inadequate ventilation, and restricted access to health care services can facilitate the transmission of TB in prisons.4,5 The prevalence of TB is estimated to be up to 100 times higher in prisons than in the general population. 6 Recent reports from 2 Malaysian prisons have shown a very high (>80\%) prevalence of latent TB infection among prisoners, irrespective of HIV status.7,8 A follow-up intensified case-finding survey in one of these prisons reported a high prevalence $(7.7 \%)$ of previously undiagnosed active TB.9 This prison is overcrowded and has a daily census of 4200 prisoners, operating at $120 \%$ of its maximum capacity of 3500.8

The high burden of TB within prison poses a threat to the general population, as the disease can be transmitted to correctional staff who are in daily close contact with prisoners. Furthermore, inmates who are incarcerated for a short period of time and who do not receive adequate treatment may transmit the disease on their return to the community.10 In a recent systematic review, a large fraction of TB cases in the general population was attributable to acquisition of TB inside prison. This has been estimated at 1 in 11 TB cases in high-income countries and 1 in 16 cases in low-/middleincome countries. 4 Another study estimated that for each $1 \%$ increase in the incarceration rate in European and Central Asian countries, there was a $0.34 \%$ increase in TB incidence in the general population.11 
Despite recent findings of high prevalence rates of both latent and active TB in Malaysian prisons, routine intensified TB screening and treatment is not currently practiced, and diagnosis of active TB remains passive. Environmental conditions, such as overcrowding and poor ventilation, further facilitate the transmission and spread of TB in these settings. The objective of this modelling study is to evaluate the impact of implementing TB treatment strategies and of improving environmental conditions in the largest Malaysian prison, considering both high (ie, average sentence length of 5 months) and low (3 years) inmate turnover rates.

\section{Methods}

Model

We constructed a deterministic population-based compartmental model of TB transmission based on previously published models.12,13 We simulated infection and reinfection, treatment of infectious individuals and latently infected individuals, death due to TB, natural recovery, and relapse (Figure 1). Our model divides the inmate population in into 5 compartments reflecting their TB status: susceptible (S), short-term latently infected (L1), long-term latently infected (L2), infectious (I), and recovered (R). Susceptible inmates acquired TB infection following contact with an actively infectious inmate at the rate $\beta \mathrm{l}$, where $\beta$ is the transmission rate that one susceptible inmate becomes infected following contact with an infectious inmate. On exposure to infectious inmates, susceptible inmates who became infected move to the short-term, latently infected compartment (L1). A proportion ( $\rho: 5 \%$ to $10 \%$ ) of latently infected individuals quickly progress at a rate of $\sigma 1$ (within the first 2 years) to the infectious stage, while the majority ( ) 1- $\rho$ progress to the long-term latent stage at a slower rate of $\sigma 2$ (within 5 years). 14 Latently infected individuals progressed to the active TB compartment at a reactivation rate of $\omega$. Once in the active stage of the disease, inmates recovered naturally at a rate of $\alpha$ or with treatment at a rate of $\alpha$ and moved into the recovered compartment. Infectious inmates (I) died from TB at a rate of $\mu 1$ and recovered individuals relapsed at a rate of $r$. Environmental factors in prison can increase the risk of infection. Hence, inmates become reinfected and return to the $L 1$ compartment at a rate of ( ) $1-f \mid \beta$. The reinfection mechanism occurs in 2 ways: (1) reinfection of recovered-stage inmates and (2) reinfection of longterm latent-stage inmates. The parameter $f$ represents reduced risk of infection due to acquired immunity from a previous infection.14-16 The parameters $\tau 1$ and $\tau 2$ represent preventive therapy initiated during the latent stage. Inmates in our model enter and exit each state at a constant rate, $\Omega$. Exiting arrows, which are represented as $\mu$, are not depicted in Figure 1. Details on the model 
equations and sources of data are in the Online Supplementary File (available in the online version of the article). Several model parameters (namely, transmission rate, prison entry/exit rate, recovery rates, and inmate turnover) could be determined based on Kajang Prison data. Further details of the quantification and justification of the transmission rate using the Wells-Riley equation for the modelling of airborne transmission and all sources of data used can be found in the Online Supplementary File. The initial conditions of the Susceptible and Recovered compartments were set based on observed prevalences in Kajang Prison, at 3\% and 4\%, respectively. In summary, the parameters transmission rate, $\beta$; rate of exit and entering prison, $\Omega$; and the recovery rates, $\tau 1, \tau 2$, and $\alpha$, are specific to the Malaysian setting; the remaining parameters were based on data from other countries. Further details can be found in the Online Supplementary File.

\section{Simulation of TB Intervention Strategies}

The first simulation adopted parameters reflecting the current environmental conditions of Kajang Prison (ie, overcrowded cell occupancy and poor ventilation; Table 1), with no interventions included. We termed this Baseline-A. Then, we ran 3 sets of simulations including the following TB interventions: isoniazid preventive therapy (IPT) provided to all latently infected inmates only (Strategy 1A); anti-TB treatment (ATBT) provided to all infectious inmates only (Strategy 2A); and both ATBT and IPT provided to all infectious and latently infected inmates (Strategy $3 \mathrm{~A}$ ). We repeated these simulations by changing the overcrowding factor (reducing the occupancy of the cell from 6 inmates [3.33 $\mathrm{m} 2$ of floor area per inmate] to 4 inmates per cell [5.40 $\mathrm{m} 2$ of floor area per inmate]) and improving the ventilation by increasing the air changes from 2 air changes per hour $(\mathrm{ACH})$ to $12 \mathrm{ACH}$ (the source of these data can be found in the Online Supplementary File). These improved conditions are referred to with the suffix "-B" in Table 1. We also repeated these simulations in a scenario where the average sentence is 3 years (vs 5 months) and refer to this scenario with the suffix "- 3 " in Table 1 . Uncertainty and sensitivity analyses are elaborated in the Online Supplementary File.

\section{Results}

\section{Simulation of TB Intervention Strategies}

Table 2 shows the impact of various strategies on the prevalence of active TB over a period of 10 years. Figures illustrating the various simulation strategies are available in the Online Supplementary File (Figures 1-4). 
With Current Environmental Conditions. Based on the current environmental conditions, if neither IPT nor ATBT is implemented (Strategy Baseline-A), the prevalence of active TB will reach a steady state of $5.77 \%$ (95\% confidence interval $[\mathrm{Cl}]=4.61-6.94)$ after 2 years, which is lower than the initial prevalence of $8 \%$ (Figure 1 in the Online Supplementary File). Implementing IPT (Strategy 1A) or ATBT (Strategy 2A) would reduce the prevalence to $5.58 \%(95 \% \mathrm{Cl}=4.60-6.72)$ and $3.28 \%(95 \% \mathrm{Cl}=$ 2.66-3.93), respectively. The combined IPT + ATBT strategy would reduce the prevalence further to $3.14 \%(95 \% \mathrm{Cl}=2.66-3.73)$. In a prison with a longer average sentence (ie, lower turnover rate), without any intervention, the prevalence of active TB was estimated to be $8.80 \%(95 \% \mathrm{Cl}=3.55-$ 15.04; Strategy Baseline-A3; Figure 2 in the Online Supplementary File); implementation of IPT alone, ATBT alone, or IPT + ATBT in this setting would reduce the prevalence of active TB to $7.99 \%$ (Strategy 1A-3), 1.94\% (Strategy 2A-3), and 1.47\% (Strategy 3A-3), respectively (Table 2). The combined intervention resulted in the lowest prevalence of active TB in both the short (5 months) and long ( 3 years) average sentence length scenarios.

Impact of Improved Environmental Conditions. In a prison setting with a high turnover of inmates (average sentence of 5 months), improving the air ventilation rate from $2 \mathrm{ACH}$ to $12 \mathrm{ACH}$ and reducing the occupancy rate from 6 to 4 inmates per cell resulted in a slight reduction of active TB prevalence in all simulations, with and without treatment strategies. In the absence of treatment (Improved-B), the prevalence of active TB decreased to $5.43 \%(95 \% \mathrm{Cl}=4.60-6.38$; Figure 3 in the Online Supplementary File). Introducing IPT (Strategy 1B) or ATBT (Strategy 2B) reduced the prevalence to $5.08 \%(95 \% \mathrm{Cl}=4.55-5.66)$ and $3.11 \%(95 \% \mathrm{Cl}=3.27-3.59)$, respectively. The combined strategy (Strategy 3B) resulted in the lowest active TB prevalence $(2.92 \%(95 \% \mathrm{Cl}=2.65$ 3.20). Improved environmental conditions resulted in only a minor reduction to the already low prevalence of active TB when the combined strategy was implemented (Table 2). However, in a prison setting with a low turnover of inmates (3 years), improved environmental conditions further reduced the prevalence of active TB to $4.15 \%(95 \% \mathrm{Cl}=2.80-6.96)$ with IPT alone (Strategy 1B-3) and $1.36 \%(95 \% \mathrm{Cl}=0.73-2.16)$ with ATBT alone (Strategy 2B-3). The combined strategy (Strategy 3B-3) resulted in a prevalence of active TB of $0.98 \%(95 \% \mathrm{Cl}=0.83-1.17$; Figure 4 in the Online Supplementary File).

\section{Discussion}

This study simulated the impact of various TB treatment and prevention strategies and modifications to environmental factors in a prison setting with a high prevalence of active and latent TB. It also 
explored the impact of inmate turnover rate on prevalence rate under the different intervention strategies. Our model suggests that, regardless of inmate turnover rate, the greatest impact on the prevalence of active TB can be achieved through provision of ATBT to infectious inmates in combination with IPT for latently infected inmates, together with a reduction of overcrowding and an improvement in air ventilation rates (Strategies 3A, 3A-3, 3B, and 3B-3). Our model also suggests that providing ATBT treatment for those infected with active TB (Strategies 2A, 2A-3, 2B, and 2B-3) alone and in combination with IPT, even in the absence of environmental improvements, would significantly reduce the prevalence of active TB over a 10-year period. Implementing IPT alone would also decrease the prevalence of active TB, but to a lesser extent as compared with ATBT alone or the combined treatment strategies.

Mathematical models of TB in a high prevalence setting have been used to evaluate the impact of treating active TB,17 using preventive therapy18,19 and of both interventions.13,20,21 However, many of these studies were conducted in open community settings. Modelling studies addressing TB in closed settings are very limited. Legrand et al,22 modelled the impact of administrative interventions on TB control in closed settings, but did not consider the impact of IPT or environmental interventions. Johnstone-Robertson et al,23 and Urrego et al,24 investigated the impact of overcrowding and poor ventilation on TB transmission in their modelling, but their analyses were limited to new infections and did not consider disease progression.

In our model, in the absence of treatment strategies, a prison with longer average sentences of 3 years (representing a low inmate turnover rate) developed a higher prevalence of active TB compared with a prison with a shorter average sentence of 5 months (high turnover rate). This finding is consistent with previous studies showing that inmates with longer sentences have a higher risk of infection because of greater exposure to infectious inmates.25,26 Reducing inmate overcrowding and enhancing quality of ventilation had a smaller impact on reducing TB transmission in prisons than treatment strategies. Without any treatment interventions, improvement of environmental conditions has little impact because inmates with preexisting comorbidities are still highly vulnerable to infection and thus progression to the infectious stage, and so contribute to the escalation of TB transmission. A study assessing risk factors of TB in 12 prisons in Brazil found that in a high-burden prison, the estimated time from infection to disease onset is around 180 days.25 In prisons with longer average sentences and poor environmental conditions, latently infected inmates would have sufficient time to progress to the actively infectious stage and consequently escalate TB transmission in the prison. 25 
Interestingly, our findings showed that the impact of treatment interventions is greater for prisons with longer average sentences compared with prisons with shorter average sentences. For example, when IPT + ATBT (Strategy 3) was implemented, the prevalence of TB was reduced by more than half, from $3.14 \%$ to $1.47 \%$ when the average sentence length was increased from 5 months to 3 years. Treatment strategies were less effective for prisons with shorter sentences because these prisons function as a queuing system: a prison has a maximum capacity-it is only able to hold a certain number of inmates at any point-and exiting inmates get replaced by new inmates who are at a high risk of being infectious because of the nature of this population.26 Releasing inmates early from a prison without implementing any TB interventions for the incoming "potential" inmates could therefore contribute to increased TB prevalence in the community over time.

One of the main factors responsible for a high rate of TB transmission in prison compared with the general population is the overcrowding, which affects ventilation. 27 Our model suggests that improving ventilation and reducing cell occupancy have a notable impact on the prevalence of active TB. However, this impact is comparatively greater when a treatment strategy is concurrently introduced and when the changes are implemented in a prison with a longer average sentence. This finding concurs with a recent study in a highly endemic Brazilian prison that found that improving ventilation reduced the TB transmission risk by $38.2 \% .24$ However, this study differed methodologically from ours in that it applied a revised Wells-Riley equation in estimating the probability of infection in the presence of one infectious individual in a cell. Our study adopted the approach of Noakes et al,28 which addressed the limitations of ventilation-based models of indoor airborne transmission by combining this approach with a classical SEIR epidemic model.

Our model has several limitations. We used a dynamic transmission model to project the impact of TB control strategies over time; the model assumes homogenous mixing, which is a potentially important oversimplification of the actual context. The airborne transmission model used was based on the even distribution of infectious particles in a room and does not account for how the probability of infection is modified by complexities, such as the distance between individual prisoners. Our study was based on data from a single prison and thus may not be representative of all prisons in Malaysia. Nevertheless, the overcrowded and poorly ventilated Kajang prison represents a common prison setting in many low- and middle-income countries. Our model therefore may be applicable to similar prisons in Malaysia with high TB prevalence.

Although our study looked at TB control measures based on treatment of active and latent infections, we did not simulate delays in initiating screening, diagnosing, and treating TB.22 Given the low prevalence of drug-resistance reported to date $(<1 \%)$, we did not consider effects of drug- 
resistance and drug-sensitive strains.29 We did not model possible IPT-related adverse events, as a systematic review found that isoniazid-induced hepatotoxicity among high-risk groups was rare. 30

Finally, in contrast to numerous prisons around the world, the HIV seroprevalence in Kajang prison is moderately low (3\%).8 We therefore did not consider the effect of HIV infection on TB. Due to the lack of local parameter estimates, the parameters for natural progression of TB disease used to inform our model additionally relied on available published studies from other countries, mainly modelling studies from developed countries in Western Europe and the United Kingdom. Estimates from these studies may differ from those for our local, higher risk population. Previous TB modelling studies in prison in Brazil and South Africa faced similar challenges, as they had to rely on studies to estimate disease progression rates.20-24,26 Our findings should be viewed as the beginning stage for further research, while at the same time highlighting the need for and potential of immediate action to address TB transmission in prisons. Without intervention, TB prevalence will remain high and contribute to the burden of TB in the prison as well as in the general population.

\section{Conclusions}

Our model demonstrated that implementation of environmental controls combined with treatment and prevention strategies can reduce the prevalence of active TB in a crowded prison setting. However, the impact is comparatively greater for prisons with a longer average sentence duration than with shorter average sentences. Theoretically, provision of treatment should be feasible given that these individuals are confined within the prison, reducing the probability of treatment nonadherence; in reality, many prisons-particularly in low- and middle-income settings-lack even basic medical care for inmates. Our simulation results suggest that ensuring adequate treatment for those infected with TB and improvements in the environmental conditions within these closed settings can greatly reduce the burden of TB in prison and, ultimately, in the general population.

\section{Authors' Note}

The data sets used during the current study are available from the corresponding author on reasonable request. All data generated or analyzed during this study are included in this article and the online supplementary file. 


\section{Declaration of Conflicting Interests}

The author(s) declare no potential conflicts of interest with respect to the research, authorship, and/or publication of this article.

\section{Funding}

The author(s) disclose receipt of the following financial support for the research, authorship, and/or publication of this article: Funding for this project was provided by the University of Malaya (University Malaya Research Grant [UMRG] RP004J-13ICT, Demographic Network Modeling of the Spread of Infectious Diseases and High Impact Research Grant HIR H-20001-E000092, Mitigation the Malaysia HIV Epidemic with a Comprehensive Research Programme).

\section{References}

1. World Health Organization. Global Tuberculosis Report 2017. Geneva, Switzerland: World Health Organization; 2017.

2. Ministry of Health Malaysia. Health Facts 2016. Putrajaya, Malaysia: Ministry of Health Malaysia, Planning Division, Health Informatics Centre; 2016.

3. World Health Organization. Malaysia. Tuberculosis profile 2013. https://extranet.who.int/sree/ Reports?op=Replet\&name=/WHO_HQ_Reports/G2/PROD/EXT/TBCountryProfile\&ISO2=my\&outt ype=pdf. Accessed November 20, 2014.

4. Baussano I, Williams BG, Nunn P, Beggiato M, Fedeli U, Scano F. Tuberculosis incidence in prisons: a systematic review. PLoS Med. 2010;7:e1000381.

5. Getahun H, Gunneberg C, Sculier D, Verster A, Raviglione M. Tuberculosis and HIV in people who inject drugs: evidence for action for tuberculosis, HIV, prison and harm reduction services. Curr Opin HIV AIDS. 2012;7:345-353.

6. Bone A, Aerts A, Grzemska M, et al; WHO Global Tuberculosis Programme; International Committee of the Red Cross. Tuberculosis Control in Prisons: A Manual for Programme Managers. Geneva, Switzerland: World Health Organization; 2000. 
7. Margolis B, Al-Darraji HA, Wickersham JA, Kamarulzaman A, Altice FL. Prevalence of tuberculosis symptoms and latent tuberculous infection among prisoners in northeastern Malaysia. Int J Tuberc Lung Dis. 2013;17:1538-1544.

8. Al-Darraji HAA, Kamarulzaman A, Altice FL. Latent tuberculosis infection in a Malaysian prison: implications for a comprehensive integrated control program in prisons. BMC Public Health. 2014;14:22.

9. Al-Darraji HA, Altice FL, Kamarulzaman A. Undiagnosed pulmonary tuberculosis among prisoners in Malaysia: an overlooked risk for tuberculosis in the community. Trop Med Int Health. 2016;21: 1049-1058.

10. PLoS Medicine Editors. The health crisis of tuberculosis in prisons extends beyond the prison walls. PLoS Med. 2010;7:e1000383.

11. Stuckler D, Basu S, McKee M, King L. Mass incarceration can explain population increases in TB and multidrug-resistant TB in European and central Asian countries. Proc Natl Acad Sci U S A. 2008;105:13280-13285.

12. Gomes GMM, Rodrigues P, Hilker FM, et al. Implications of partial immunity on the prospects for tuberculosis control by post-exposure interventions. J Theor Biol. 2007;248:608-617.

13. Silva CJ, Torres DFM. Optimal control for a tuberculosis model with reinfection and postexposure interventions. Math Biosci. 2013;244:154-164.

14. Vynnycky E, Fine PE. The natural history of tuberculosis: the implications of age-dependent risks of disease and the role of reinfection. Epidemiol Infect. 1997;119:183-201.

15. Dowdy DW, Dye C, Cohen T. Data needs for evidence-based decisions: a tuberculosis modeler's "wish list." Int J Tuberc Lung Dis. 2013;17:866-877.

16. Sutherland I, Svandova E, Radhakrishna S. The development of clinical tuberculosis following infection with tubercle bacilli. 1. A theoretical model for the development of clinical tuberculosis following infection, linking from data on the risk of tuberculous infection and the incidence of clinical tuberculosis in the Netherlands. Tubercle. 1982;63:255-268.

17. Dye C, Garnett GP, Sleeman K, Williams BG. Prospects for worldwide tuberculosis control under the WHO DOTS strategy. Directly observed short-course therapy. Lancet. 1998;352:1886-1891. 
18. Mushayabasa S, Bhunu CP. Modeling the impact of early therapy for latent tuberculosis patients and its optimal control analysis. J Biol Phys. 2013;39:723-747.

19. Ziv E, Daley CL, Blower SM. Early therapy for latent tuberculosis infection. Am J Epidemiol. 2001;153:381-385.

20. Basu S, Andrews JR, Poolman EM, et al. Prevention of nosocomial transmission of extensively drugresistant tuberculosis in rural South African district hospitals: an epidemiological modelling study. Lancet. 2007;370:1500-1507.

21. Bhunu CP, Garira W, Mukandavire Z, Zimba M. Tuberculosis transmission model with chemoprophylaxis and treatment. Bull Math Biol. 2008;70:1163-1191.

22. Legrand J, Sanchez A, Le Pont F, Camacho L, Larouze B. Modeling the impact of tuberculosis control strategies in highly endemic overcrowded prisons. PLoS One. 2008;3:e2100.

23. Johnstone-Robertson S, Lawn SD, Welte A, Bekker LG, Wood R. Tuberculosis in a South African prison-a transmission modelling analysis. S Afr Med J. 2011;101:809-813.

24. Urrego J, Ko Al, da Silva Santos Carbone A, et al. The impact of ventilation and early diagnosis on tuberculosis transmission in Brazilian prisons. Am J Trop Med Hyg. 2015;93:739-746.

25. Ada CS, Paiao DS, Sgarbi RV, et al. Active and latent tuberculosis in Brazilian correctional facilities: a cross-sectional study. BMC Infect Dis. 2015;15:24.

26. Basu S, Stuckler D, McKee M. Addressing institutional amplifiers in the dynamics and control of tuberculosis epidemics. Am J Trop Med Hyg. 2011;84:30-37.

27. World Health Organization. WHO policy on TB Infection Control in Health-Care Facilities, Congregrate Settings and Households. Geneva, Switzerland: World Health Organization; 2009.

28. Noakes CJ, Beggs CB, Sleigh PA, Kerr KG. Modelling the transmission of airborne infections in enclosed spaces. Epidemiol Infect. 2006;134:1082-1091.

29. Ministry of Health. Clinical Practice Guidelines. Management of Tuberculosis. 3rd ed. Kuala Lumpur, Malaysia: Ministry of Health; 2012.

30. Bliven EE, Podewils $L J$. The role of chronic hepatitis in isoniazid hepatotoxicity during treatment for latent tuberculosis infection. Int J Tuberc Lung Dis. 2009;13:1054-1060. 